\title{
Dark-field X-ray imaging of unsaturated water transport in porous materials
}

\author{
F. Yang, ${ }^{1,2, a)}$ F. Prade,${ }^{3}$ M. Griffa, ${ }^{1, a)}$ I. Jerjen, ${ }^{1}$ C. Di Bella,${ }^{1,2}$ J. Herzen, ${ }^{3}$ A. Sarapata, ${ }^{3}$ \\ F. Pfeiffer, ${ }^{3}$ and P. Lura ${ }^{1,2}$ \\ ${ }^{1}$ Empa, Swiss Federal Laboratories for Materials Science and Technology, Dübendorf 8600, Switzerland \\ ${ }^{2}$ Institute for Building Materials (IfB), ETH Zurich, Zürich 8093, Switzerland \\ ${ }^{3}$ Physik-Department \& Institut für Medizintechnik, Technische Universität München, Garching, Germany
}

(Received 23 August 2014; accepted 7 October 2014; published online 17 October 2014)

\begin{abstract}
We introduce in this Letter an approach to X-ray imaging of unsaturated water transport in porous materials based upon the intrinsic X-ray scattering produced by the material microstructural heterogeneity at a length scale below the imaging system spatial resolution. The basic principle for image contrast creation consists in a reduction of such scattering by permeation of the porosity by water. The implementation of the approach is based upon X-ray dark-field imaging via Talbot-Lau interferometry. The proof-of-concept is provided by performing laboratory-scale dark-field X-ray radiography of mortar samples during a water capillary uptake experiment. The results suggest that the proposed approach to visualizing unsaturated water transport in porous materials is complementary to neutron and magnetic resonance imaging and alternative to standard X-ray imaging, the latter requiring the use of contrast agents because based upon X-ray attenuation only. (C) 2014 AIP Publishing LLC. [http://dx.doi.org/10.1063/1.4898783]
\end{abstract}

In porous materials, e.g., several building materials, water ingress may cause unwanted swelling ${ }^{1}$ or high degrees of pore saturation that might result in damage upon freezing. ${ }^{2}$ In addition, water may carry harmful ions that, for example, may induce salt precipitation in the pores with consequent cracking due to crystal growth, ${ }^{3,4}$ expansive reactions, ${ }^{5}$ or reinforcement corrosion..$^{6}$

Water sorptivity is a material property characterizing the tendency to absorb water by capillarity. ${ }^{7}$ This property is usually measured gravimetrically, ${ }^{7}$ which allows precise measurements of the total amount of absorbed water. The determination of the water front evolution and of the water content profiles from sorptivity data is less accurate and depends on a number of assumptions resulting in substantial errors. ${ }^{8}$ Independent methods for assessing the distance of penetration of the wetting front include ${ }^{1} \mathrm{H}$ nuclear magnetic resonance imaging ${ }^{9}$ (MRI), neutron radiography ${ }^{10}(\mathrm{NR})$, and $\mathrm{X}$-ray radiography ${ }^{11}(\mathrm{XR})$. The latter is widely available, contrary to NR and MRI, and would offer the additional advantage of assessing microstructural features in the porous material (e.g., cracks, pores in the range of $10-100 \mu \mathrm{m}$, and density gradients) which might affect water sorption. Such assessment is not feasible with NR and MRI, due to their lower spatial resolution. Standard XR is based upon the measurement of X-ray linear attenuation coefficients.

Compared with the porous matrix, especially for building materials and geomaterials, water attenuates X-rays only poorly. Thus, standard XR leads to poorly resolved and very noisy water profiles, unless water is mainly localized in very large (mm-scale) pores. This problem can be alleviated by adding a contrast agent with high $\mathrm{X}$-ray attenuation to the water, for example, lead nitrate ${ }^{12}$ or potassium iodide. ${ }^{13}$ However, sorptivity experiments with pure water are preferred, since the contrast agent influences the properties

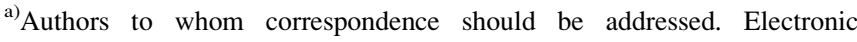
addresses: fei.yang@empa.ch and michele.griffa@empa.ch.
}

of the solution and may react with or be adsorbed onto the porous material.

In this work, we propose an approach to X-ray imaging of unsaturated water transport in a porous (building) material. The approach exploits the multiple X-ray scattering produced by the intrinsic microstructural heterogeneity of the porous material, leading to small angle X-ray scattering (SAXS) emerging from the sample.

A radiographic image with contrast based upon multiple $\mathrm{X}$-ray scattering, also called dark-field image, visualizes the 2D spatial distribution of the projected scattering strength of the material itself. ${ }^{14}$ The scattering strength is higher where the microstructure is more heterogeneous, i.e., where the electron density varies on a length scale which is below the spatial resolution of the imaging instrument. ${ }^{14-16}$

The basic principle on which our approach is based, consists in the fact that when water fills the pores, reduces the scattering strength of the material because the gradients in the X-ray refractive index spatial distribution are decreased, thus the $\mathrm{X}$-ray multiple scattering. ${ }^{17}$ The signal of the dark-field image is built upon decreases as well, consequently creating a significant contrast between unsaturated and saturated regions. ${ }^{18}$

The chosen X-ray dark-field imaging method is based upon the Talbot-Lau interferometry (TLI), allowing simultaneously measuring the $\mathrm{X}$-rays attenuation, refraction, and scattering by the sample. This is achieved by analyzing a periodic X-ray interference pattern which changes due to the $\mathrm{X}$-ray-sample interactions. ${ }^{19}$

The output from a single TLI measurement thus consists in three radiographs, i.e., linear projections, of three different physical parameters: the X-ray linear attenuation coefficient $\mu$, related with the imaginary part $\beta$ of the $\mathrm{X}$-ray refractive index $n$, the partial derivative of the decrement $\delta$, with respect to 1 , of the real part of $n$, and the X-ray scattering coefficient $\xi^{20,21}$ The three types of radiographs are characterized by different contrast to different materials and 
micro-structural features. The simultaneous acquisition of three radiographs with different contrasts represents a key advantage of this technique, together with the possibility of implementing it with standard laboratory X-ray sources. $^{19,22}$

In order to prove the feasibility and the potential of $\mathrm{X}$-ray dark-field imaging of water in a porous material, we designed a water capillary uptake experiment with mortar samples based on radiography (2D imaging) with a TLI.

Liquid capillary uptake consists in the unsaturated flow driven by capillary forces and progressing from one or more boundaries in contact with the liquid towards the bulk. We chose to image the spatial-temporal distribution of water absorbed into the samples in order to evaluate both the contrast and the temporal resolutions of the dark-field X-ray radiography method.

We chose mortar as a test material because of the relevance of water transport in several processes related with its microstructural development, during cement hydration, and with its durability.

We prepared three samples with rectangular shape and size $10 \times 20 \times 2 \mathrm{~mm}^{3}$ (width, height, and thickness, respectively). The thickness is measured along the propagation axis of the X-rays. The three samples were made according to the same mix design. ${ }^{23}$ After cutting, the three samples were dried in an oven at $50{ }^{\circ} \mathrm{C}$ for $48 \mathrm{~h}$. Afterwards, two of them were further thermally treated (pre-conditioning). One sample (C_200D) was pre-conditioned at $200^{\circ} \mathrm{C}$ for $1 \mathrm{~h}$, in order to induce thermal micro-cracking. ${ }^{24}$ Another sample (C_120D) was pre-conditioned at $120^{\circ} \mathrm{C}$ for $3 \mathrm{~h}$. The third sample (C_U) underwent no thermal treatment.

The three samples were expected to differ in terms of their liquid sorptivity, as it relates to the amount of dehydration and thermal micro-cracking (the higher the pre-conditioning temperature, the larger the amount of microcracks, the larger the sorptivity). After the pre-conditioning, a $0.07 \mathrm{~mm}$ thick polyimide film tape was applied to each sample's lateral surface, except for the bottom, which was put in contact with water. This tape provides high transparency to $\mathrm{X}$-rays and reduces the evaporation from the lateral surfaces. Given the boundary conditions and the large aspect ratios of the samples, the water capillary uptake can be approximately described as a mass transfer process preferentially along the height of the samples.

The experiment consisted in the following protocol. A plastic container with de-mineralized water was introduced at the bottom of the field of view (FOV) of the TLI. Ten X-ray radiographs were initially acquired before introducing the samples in the FOV. These radiographs were used in the image processing to retrieve the three types of radiographs (flat-field correction stage of the phase-stepping protocol ${ }^{19,22}$ ).

The three samples were fixed side by side from their top to a holder, they were introduced in the FOV and moved down in order to submerge their bottom part into water for about $3 \mathrm{~mm}$. Immediately after immersion, X-ray radiographs were acquired continuously, following the phase stepping protocol, ${ }^{19,22}$ in order to track the capillary uptake. The overall experiment lasted about $13 \mathrm{~h}$. During the first hour, the images were taken approximately every $11 \mathrm{~s}$, while in the following eleven hours every $131 \mathrm{~s}$. A higher sampling frequency was required during the first hour in order to improve the capturing of the initial stages of the uptake. Despite such higher frequency, the very initial stages were missed because of the dead time occurring between the immersion of the samples' bottom into water and the actual start of the radiography acquisition. All the details about the TLI configuration, the phase stepping protocol for the multi-contrast X-ray radiograph retrieval from the raw radiographs and the associated image processing are reported in Sec. II of Ref. 23.

Figure 1 shows a selection of dark-field (insets (a) and (b)) and attenuation (insets (c) and (d)) radiographs at two different time instants ((a) and (c) at the beginning, (b) and (d) at the end) of the capillary uptake experiment. The pixel value in the dark-field radiographs corresponds to the cumulative value (linear projection) of $\xi$ (expressed in units of $10^{-12}$ ), thus proportional to the scattering cumulative strength. The pixel value in the attenuation radiographs corresponds to the cumulative value of $\mu$ (in units of $\mathrm{cm}^{-1}$ ). ${ }^{23}$

The water wetting front is almost invisible in the attenuation radiographs. Only an average pixel value increase can be faintly, visually perceived with passing time (from inset (c) to (d)). A better perception of changes, even though still subtle, is achieved by looking at the entire time series of the attenuation radiographs. See Ref. 23 for the movie

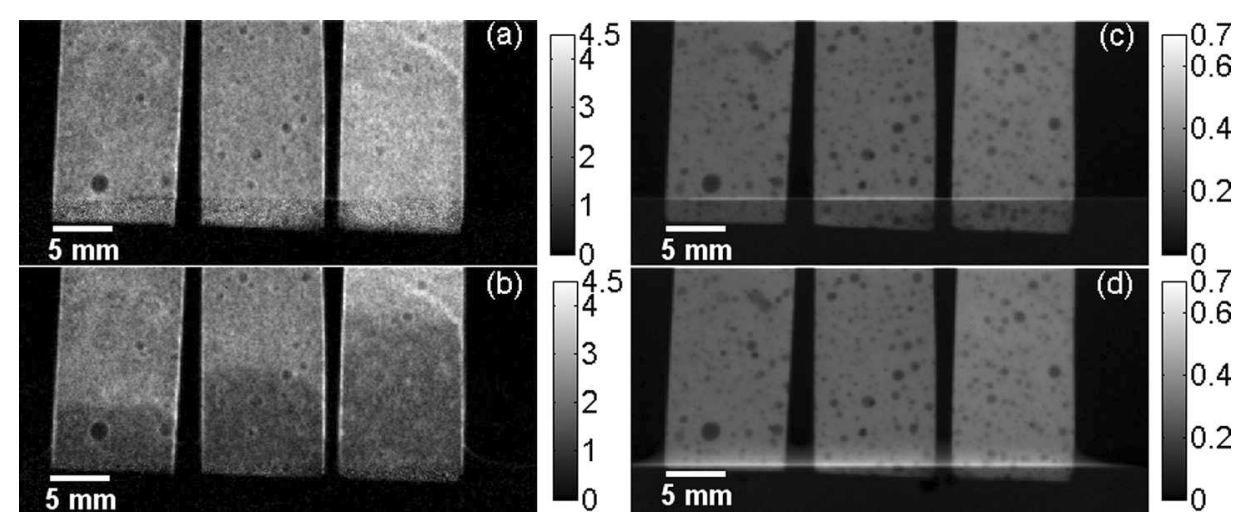

FIG. 1. X-ray radiographs at two different time instants during the capillary uptake, increasing from the top to the bottom. (a) and (b) Dark-field radiographs. Brighter pixel values correspond to larger scattering strength. (c) and (d) Attenuation radiographs. Brighter pixel values correspond to larger X-ray attenuation. Radiographs in the same row refer to the same time instant. The samples are C_U (undamaged), C_120D (thermally damaged at $120{ }^{\circ} \mathrm{C}$ ), and C_200D (thermally damaged at $200^{\circ} \mathrm{C}$ ), respectively, from left to right. The bright horizontal lines visible towards the bottom in (c) and (d) are artifacts created by evaporation from the water surface during the experiment. See Sec. III of Ref. 23 for additional explanation about the source of these artifacts. 
containing the time series of both the attenuation radiographs (top row) and the dark-field (bottom row) radiographs.

The wetting front and the wetted part of each sample are instead well distinguished in the dark-field radiographs (insets (a) and (b)). In those images, the wetted regions appear darker than the non-wetted ones, confirming our initial hypothesis about the scattering reduction by water permeating the material porosity.

The capillary uptake in all the samples is characterized by a wetting front proceeding towards the top and not completely uniform along the sample width. The latter feature is due to (a) the microstructural inhomogeneity of the porous material, specifically the presence of large spherical pores created by air entrapped during the mixing of the mortar, and to (b) boundary effects. Such features can be better appreciated in Fig. 2, where the wetting front is plotted as a red line (color online) on top of a central region of interest (ROI) of the last attenuation radiograph of each sample ((a) C_U, (b) C_120D, and (c) C_200D, respectively). The wetting front has been extracted from the dark-field radiographs according to the image processing algorithms described in Sec. IV of Ref. 23.

Different lines in Fig. 2 correspond to different time instants, the initial one being about $t=2.9 \mathrm{~min}$, thus in the initial stage of the process, and the time gap between two successive lines corresponding to 25 radiographic sampling time steps. Figure 2 shows, for every sample, a significant curvature of the wetting front in correspondence of the largest pores. In addition, the wetting front tracing in Fig. 2 confirms the increase of sorptivity with the thermal preconditioning of the samples: the inter-distance between two temporally successive fronts increases on average from inset (a) to inset (c).

In order to obtain a more quantitative characterization of the change in contrast between a wetted region and a non-
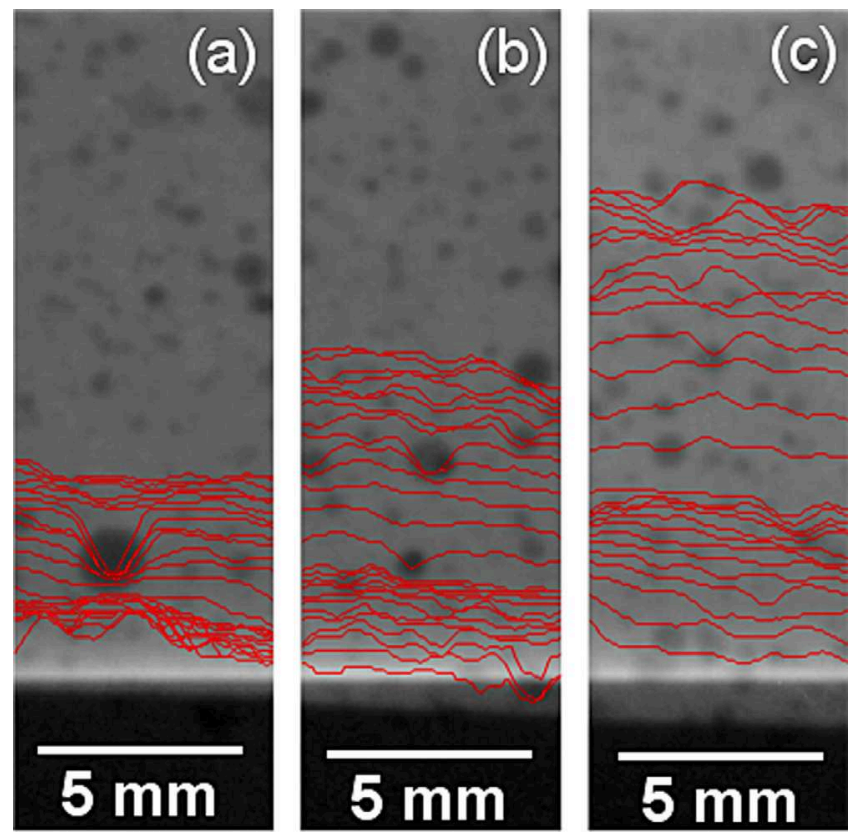

FIG. 2. Wetting front positions (red lines) overlaid on top of a central region of interest of the last attenuation radiograph, for the three different samples: (a) C_U, (b) C_120D, (c) C_200D. For each sample, the first wetting front is plotted at approximately time $t=2.9 \mathrm{~min}$. The time gap between two successive fronts is 25 temporal points. wetted one, both for the dark-field and the attenuation radiographs, we calculated the contrast-to-noise ratio (CNR) given a ROI that is permeated by water and one that remains at any time dry. At a given time instant, for a given radiograph type and a given sample, the CNR was calculated as

$$
C N R=\left|S_{w e t}-S_{d r y}\right| / \sqrt{\sigma_{w e t}^{2}+\sigma_{d r y}^{2}},
$$

where $S_{\text {wet }}$ is the average pixel value of the image within the wetted ROI, $S_{d r y}$ the corresponding average value for the non-wetted ROI and $\sigma_{w e t}$ and $\sigma_{d r y}$ the pixel value standard deviation for the two ROIs, respectively. For a given image type and sample, a larger CNR after a wetting step indicates more contrast brought by water wetting. Thus, the image type with larger increases in the CNR during wetting allows for better visualization of the temporal changes in the water distribution.

We summarize here the results for the CNR analysis. Section V of Ref. 23 contains tables with the numerical values for each sample and for each radiograph type.

For the attenuation radiographs, the CNR decreases with time for all the samples (except for sample C_120D at time $t_{1}$ ) with a relative change between time $t_{0}$ and $t_{2}$ of the order of $30 \%-50 \%$. A small increase would be expected instead, due to the small increase of the pixel value for the wetted ROI. However, the noise level increases as well in the wetted region, while it remains constant for the dry one. The noise increase mirrors the small but highly heterogeneously distributed increase in the X-ray attenuation brought by the absorbed water.

On the contrary, the CNR increases significantly with time for the dark-field radiographs (relative change between time $t_{0}$ and $t_{2}$ of the order of $500 \%-1000 \%$ ), confirming the visible contrast in Fig. 1. We notice that the CNR value is slightly dependent upon the choice of the ROIs. However, its temporal evolution with wetting follows the same trend independently of the ROI. Results for other sets of ROIs are reported in Ref. 23.

The results reported here prove the feasibility of imaging with high contrast pure water in a porous material by exploiting X-ray scattering. This approach offers a significant number of advantages over neutron or magnetic resonance imaging.

The first advantage consists in the greater accessibility of instruments implementing X-ray dark-field imaging. All the techniques proposed for such imaging at the laboratory scale $^{19,25}$ are based upon X-ray optical components that can be added to existing laboratory instruments. This feature provides them the potential for systematic studies requiring frequently repeated, time-lapsed measurements. Such studies are not always feasible with neutron imaging because of the limited number of available facilities and access to them. Magnetic resonance imaging facilities are more accessible than neutron ones. However, they still do not achieve the same spatial and temporal resolution available by X-ray imaging.

As mentioned before, TLI provides three different images. This multi-contrast imaging mode represents a second advantage over magnetic resonance imaging of water transport in porous materials: the information about the 
water spatial distribution from the dark-field images can be complemented by the porous matrix microstructure information contained in the attenuation and dark-field images themselves. In the dark field radiographs, regions with larger microstructural heterogeneity are better highlighted, before water permeation, Fig. 1(a), even though the microstructural heterogeneity itself, e.g., the porosity, is not completely resolved. ${ }^{26,27}$ On the contrary, the attenuation radiographs allow resolving the pores with size much larger than the spatial resolution of the instrument, e.g., the spherical air voids, Fig. 1(c).

The complementarity of information is extremely important for investigating how structural features of the porous material, such as cracks with different widths, affect the transport process. The use of another approach, differential (X-ray) radiography, where radiographs are normalized by a first radiograph when the sample is completely dry, ${ }^{28}$ allows visualizing only the temporal changes in the water distribution, thus no correlations between the micro-structure and the water transport.

In general, one can foresee a significant impact of laboratory-scale X-ray dark-field imaging on studying water transport in porous geo-materials. ${ }^{29,30}$ Another field that could benefit from this approach is the development of hydrogen-based fuel cells. Water transport in polymer electrolyte membrane fuel cells could be studied more continuously during their service life, for characterizing the correlations between the transport processes and the cells' microstructural evolution, hence improving their water management and their durability. ${ }^{31-33}$

In order to fully assess and exploit the potential of X-ray dark-field imaging of unsaturated water transport in porous materials, further investigations and developments are needed in two main directions.

On the one hand, it is necessary to extensively characterize the contrast produced in dark-field images by water uptake as a function of the saturation degree and the microstructure heterogeneity, specifically the pore (and crack, when present) size distribution. Quantitative relations between the pixel value of dark-field radiographs obtained with the TLI and the porous material microstructural heterogeneity have already been proposed via different models of wave propagation in random media. ${ }^{34,35}$ Among these parameters there are correlation length and variance of the random microstructure and other parameters indirectly related with the shape and size distribution of microstructural features as grain, voids, and bubbles. ${ }^{34-36}$ The effectiveness of our approach to imaging water is expected to be larger in materials with narrower pore size distribution and within a range below or close to the spatial resolution of the instrument used. In those conditions, scattering is expected to be stronger. At the same time, capillary water uptake is also expected to be more efficient, due to larger capillary forces involved, leading to higher reduction in scattering, thus more contrast in the dark field images. However, this physical assumption needs to be carefully and systematically validated.

On the other hand, it is necessary to decrease the measurement time needed for the retrieval of the three types of radiographs in order to (a) allow tracking fast transport processes and (b) allow the acquisition of radiographs at different orientation angles of the sample for performing a tomographic, i.e., 3D, multi-contrast reconstruction. Different approaches have been already proposed and tested for achieving such goal. ${ }^{37-40}$

To summarize, we propose in this work an approach to $\mathrm{X}$-ray imaging of unsaturated water transport in porous materials exploiting the intrinsic X-ray scattering produced by the spatially heterogeneous microstructure of the material. Water uptake leads to a decrease in scattering, bringing high contrast between wetted and non-wetted regions. We provide a proof of concept based upon the Talbot-Lau interferometer, used for retrieving 2D images (radiographs) based on scattering (dark-field) contrast. The measurements were continuously performed during a water uptake experiments with mortar samples. The results confirm the validity of the approach and its potential for systematic, laboratory-scale imaging of water transport in diverse porous materials.

This work was performed within the framework of the Helmholtz Virtual Institute for New X-ray analytic Methods in Materials science (VI-NXMM), with technical support from the Institute of Microstructure Technology of the Karlsruhe Institute of Technology. We acknowledge financial support by the Swiss National Science Foundation (Project No. 200021_143782), the DFG Cluster of Excellence Munich-Centre for Advanced Photonics (MAP), the DFG Gottfried Wilhelm Leibniz Program and the European Research Council (ERC, FP7, StG 240142), beyond that of the VI-NXMM. We would like to thank Paul Diémoz, Rolf Kaufmann, and Veerle Cnudde for inspiring discussions about dark-field X-ray imaging and differential X-ray radiography and the anonymous reviewer for insightful comments that helped in improving the analysis of the results.

${ }^{1}$ D. Derome, M. Griffa, M. Koebel, and J. Carmeliet, J. Struct. Biol. 173(1), 180-190 (2011).

${ }^{2}$ W. Li, M. Pour-Ghaz, J. Castro, and J. Weiss, J. Mater. Civ. Eng. 24(3), 299-307 (2012).

${ }^{3}$ N. Tsui, R. J. Flatt, and G. W. Scherer, J. Cul. Heritage 4(2), 109-115 (2003).

${ }^{4}$ H. Derluyn, M. Griffa, D. Mannes, I. Jerjen, J. Dewanckele, P. Vontobel, A. Sheppard, D. Derome, V. Cnudde, E. Lehmann, and J. Carmeliet, J. Building Phys. 36(4), 353-374 (2013).

${ }^{5}$ A. Leemann and R. Loser, Cem. Concr. Compos. 33(1), 74-83 (2011).

${ }^{6}$ A. Michel, P. V. Nygaard, and M. R. Geiker, Corros. Sci. 72, 26-34 (2013).

${ }^{7}$ C. Hall, Mag. Concr. Res. 41(147), 51-61 (1989).

${ }^{8}$ D. Lockington, J. Y. Parlange, and P. Dux, Mater. Struct. 32(5), 342-347 (1999).

${ }^{9}$ T. A. Carpenter, E. S. Davies, C. Hall, L. D. Hall, W. D. Hoff, and M. A. Wilson, Mater. Struct. 26(5), 286-292 (1993).

${ }^{10} \mathrm{H}$. Justnes, K. Bryhn-Ingebrigtsen, and G. O. Rosvold, Adv. Cem. Res. 6(22), 67-72 (1994)

${ }^{11}$ G. Sant, A. Eberhardt, D. Bentz, and J. Weiss, J. Mater. Civ. Eng. 22(3), 277-286 (2010).

${ }^{12}$ A. Pourasee, A. Peled, and J. Weiss, J. Mater. Civ. Eng. 23(8), 1227-1238 (2011).

${ }^{13}$ E. Franzoni, G. Graziani, E. Sassoni, G. Bacilieri, M. Griffa, and P. Lura, "Solvent-based ethyl silicate for stone consolidation: influence of the application technique on penetration depth, efficacy and pore occlusion," Mater. Struct. (published online).

${ }^{14}$ H. N. Chapman, C. Jacobsen, and S. Williams, Ultramicroscopy 62, 191-213 (1996).

${ }^{15}$ L. E. Levine and G. G. Long, J. Appl. Cryst. 37, 757-765 (2004).

${ }^{16}$ G. R. Morrison and M. T. Browne, Rev. Sci. Instrum. 63(1), 611-614 (1992). 
${ }^{17}$ F. Zhang, G. G. Long, L. E. Levine, J. Ilavsky, and P. R. Jemian, J. Appl. Cryst. 41, 416-427 (2008).

${ }^{18}$ I. Jerjen, V. Revol, C. Kottler, T. Luethi, U. Sennhauser, R. Kaufmann, and $\mathrm{C}$. Urban, in Proceedings of the Fachtagung Industrielle Computertomographie (2010), pp. 271-276.

${ }^{19}$ F. Pfeiffer, M. Bech, O. Bunk, P. Kraft, E. F. Eikenberry, C. Brönnimann, C. Grünzweig, and C. David, Nat. Mater. 7(2), 134-137 (2008).

${ }^{20}$ Z.-T. Wang, K.-J. Kang, Z.-F. Huang, and Z.-Q. Chen, Appl. Phys. Lett. 95(9), 094105 (2009).

${ }^{21}$ M. Bech, O. Bunk, T. Donath, R. Feidenhans'1, C. David, and F. Pfeiffer, Phys. Med. Biol. 55(18), 5529-5539 (2010).

${ }^{22}$ F. Pfeiffer, M. Bech, O. Bunk, T. Donath, B. Henrich, P. Kraft, and C. David, J. Appl. Phys. 105(10), 102006 (2009).

${ }^{23}$ See supplementary material at http://dx.doi.org/10.1063/1.4898783 for a description of the sample preparation and of the Talbot-Lau interferometer used, for the image processing used to determine the wetting fronts and for the contrast-to-noise ratio analysis.

${ }^{24}$ Y. F. Fu, Y. L. Wong, C. A. Tang, and C. S. Poon, Cem. Concr. Compos. 26(2), 99-111 (2004).

${ }^{25}$ M. Endrizzi, P. C. Diemoz, T. P. Millard, J. Louise Jones, R. D. Speller, I. K. Robinson, and A. Olivo, Appl. Phys. Lett. 104(2), 024106 (2014).

${ }^{26}$ V. Revol, I. Jerjen, C. Kottler, P. Schütz, R. Kaufmann, T. Lüthi, U. Sennhauser, U. Straumann, and C. Urban, J. Appl. Phys. 110(4), 044912 (2011).

${ }^{27}$ I. Jerjen, V. Revol, A. J. Brunner, P. Schuetz, C. Kottler, R. Kaufmann, T. Luethi, G. Nicoletti, C. Urban, and U. Sennhauser, Polym. Test. 32(6), 1094-1098 (2013).
${ }^{28}$ V. Cnudde, M. Dierick, J. Vlassenbroeck, B. Masschaele, E. Lehmann, P. Jacobs, and L. Van Hoorebeke, Nucl. Instrum. Methods Phys. Res., Sect. B 266, 155-163 (2008).

${ }^{29}$ B. Berkowitz, Adv. Water Resour. 25, 861-884 (2002).

${ }^{30}$ D. Wildenschild and A. P. Sheppard, Adv. Water Resour. 51, 217-246 (2013).

${ }^{31}$ A. Bazylak, Int. J. Hydrogen Energy 34, 3845-3857 (2009).

${ }^{32}$ R. Flückiger, F. Marone, M. Stampanoni, A. Wokaun, and F. N. Büchi, Electrochim. Acta 56(5), 2254-2262 (2011).

${ }^{33}$ P. Oberholzer, P. Boillat, R. Siegrist, A. Kaestner, E. H. Lehmann, G. G. Scherer, and A. Wokaun, Electrochem. Commun. 20, 67-70 (2012).

${ }^{34}$ W. Yashiro, Y. Terui, K. Kawabata, and A. Momose, Opt. Express 18(16), 16890-16901 (2010).

${ }^{35}$ S. K. Lynch, V. Pai, J. Auxier, A. F. Stein, E. E. Bennett, C. K. Kemble, X. Xiao, W.-K. Lee, N. Y. Morgan, and H. Harold Wen, Appl. Opt. 50(22), 4310-4319 (2011).

${ }^{36}$ A. Malecki, G. Potdevin, and F. Pfeiffer, Europhys. Lett. 99(4), 48001 (2012).

${ }^{37}$ P. C. Diemoz, P. Coan, I. Zanette, A. Bravin, S. Lang, C. Glaser, and T. Weitkamp, Opt. Express 19(3), 1691-1698 (2011).

${ }^{38}$ N. Bevins, J. Zambelli, K. Li, Z. Qi, and G.-H. Chen, Med. Phys. 39(1), 424-428 (2012).

${ }^{39}$ T. Gaass, G. Potdevin, M. Bech, P. B. Noël, M. Willner, A. Tapfer, F. Pfeiffer, and A. Haase, Europhys. Lett. 102(4), 48001 (2013).

${ }^{40}$ Y. Ge, K. Li, J. Garrett, and G.-H. Chen, Opt. Express 22(12), $14246-14252$ (2014). 